\title{
Adsorption of decorated nanoparticles on a liquid crystalline polymer brush: molecular dynamics study
}

\author{
Slyusarchuk A. Y. ${ }^{1}$, Yaremchuk D. L. ${ }^{2}$, Ilnytskyi J. M. ${ }^{1,2}$ \\ ${ }^{1}$ Lviv Polytechnic National University, \\ 12 S. Bandera Str., 79013, Lviv, Ukraine \\ ${ }^{2}$ Institute for Condensed Matter Physics of the National Academy of Sciences of Ukraine, \\ 1 Svientsitskii Str., 79011, Lviv, Ukraine
}

(Received 25 March 2020; Accepted 16 June 2020)

\begin{abstract}
We consider a solution of nanoparticles in a pore with one of its walls being a liquid crystalline polymer brush. Both nanoparticles' ligands and the brush molecules side chains contain the same liquid crystalline groups. The system is studied using the molecular dynamics simulations. At both cases of a low and high brush density, the aggregation between the pairs of nanoparticles in a bulk and between the brush molecules prevail. However, we found a specific brush density when the nanoparticles are adsorbed more readily on a brush than aggregate in a bulk. A set of density profiles as well as the dynamical properties of nanoparticles are analysed in all cases considered.
\end{abstract}

Keywords: nanoparticles, liquid crystals, polymer brush, adsorption.

2010 MSC: $82 \mathrm{C} 05,82 \mathrm{C} 43$

DOI: $10.23939 / \mathrm{mmc} 2020.02 .207$

\section{Introduction}

Physical and chemical properties of nanoparticles (NP) are important in a view of their optical, magnetic, electronic and catalytic applications [1-3]. Their properties differ essentially from that of bulk materials due to quantum confinement effects and high surface-to-volume ratio. These properties, however, are weakened with the NPs aggregation and with an increase of the particle size. To prevent such demise, several solutions exist, namely, decoration of NPs via polymer ligands, incorporating them into the branched polymer structures or microgels, etc. We will concentrate here on the first in the list of these approaches. Decoration of NPs enables much more control over the NPs aggregation/deaggregation, gelation, adsorption, fine-tuning of their interaction with the surfaces and with a solvent. Many types of a NP core is currently used, typical examples are: metallic cores of Au, Ag, Rh, $\mathrm{Pd}$, Pt, Ir and Os [4-7], as well as the cores of hyper-branched polymers, aromatic compounds of discotic shape, porphyrin, fullerenes, etc. [8-14]. Among metal-based cores, probably the most common examples of decorated NP are gold-core nanoparticles, due to their important applications in heterogeneous catalysis $[7,15,16]$, the surface-plasmon-resonance-based applications [6], medical diagnostics and therapeutics $[17,18]$.

The state of decorated NPs in solution depends on the chemical properties of environment [19-22]; the type of polymer ligand, e.g. homopolymer $[8,23,24]$ or co-polymer $[25,26]$; the presence of specific functional groups, e.g. the liquid crystalline (LC) ones or chromophoric [27-30]; as well as the possibility to arrange the ligands as specific spatial patches [8,31-35]. All these factors allow one to turn decorated NPs into micro-blocks for specific supramolecular bulk assembly. Another way to control behaviour of NPs is by functional or structured surfaces [36], one example is a polymer brush, the details of synthesis is given in e.g. Refs. [37] and [38]. The interaction between the NPs and the brush wall can be made explicit if both contain compatible chemical groups that interact in a spatially-resolved way. Following some of the earlier papers [35,39-43], we will study the NPs decorated by LC groups here. 
Therefore, the natural choice for a compatible functional surface is a LC polymer brush built-up of a polymer chains of a main-chain [44] or side-chain [45] architecture. We will focus on a latter case only.

On a general note, a LC polymer brush is a collection of the LC polymers that may self-assemble into various LC phases (e.g. nematic [46]) depending on their density and the temperature. Grafting of their ends to a wall provides a constraint that stabilizes layered, e.g. smectic, phases. Several chemical techniques can be utilised to fabricate the LC polymer brush: (i) using the preformed polymer chains ("grafting-to"); (ii) surface initiated polymerization ("grafting-from"); and polymerization using surface attached monomers ("grafting-through") [47]; as well as spin-casting. The important aspect of such brush is the orientation of its LC groups, which can typically be planar or homeotropic with respect to the wall, and can be achieved by the use of appropriate preparation method [47]. Another key factor is the density of a polymer brush, as it typically undergoes the transition from a "mushroom" state at low density to the dense brush state at high density. The latter exhibits unique properties because of the stretched chain conformations, and dense LC polymer brushes can be prepared via e.g. surface segregation and self-assembly of diblock LC polymer molecules [48]. Another wide and technologically important class of LC polymer brushes are photo-sensitive brushes that contain chromophoric, e.g. azobenzene groups [49]. Such brush changes its effective height and roughness when illuminated by an uniform beam of specific wavelength [50], whereas the spatially-modulated illumination pattern leads to formation of a surface relief on it [49].

If both NPs and a brush coexist in one system, then several NP-brush mutual arrangements are possible: (i) NPs and brush phase separate; (ii) NPs are adsorbed on a surface of a brush and form a $2 D$ structure there; (iii) NPs may be adsorbed by a bulk of a brush and arrange into a $3 D$ structure; for more detailed review, see Refs. [51]. The fine details of these respective $2 D$ or $3 D$ structures depend on many factors such as brush composition and density, type of solvent, etc. In particular, for strong NP-polymer interactions, an ordered $2 D$ array is only formed at particular brush density, whereas at higher or lower density, the distribution of NPs demonstrate large fluctuations in space and the packing order is reduced. For weak NP-polymer interactions, the NPs arrange into a hexagonal array on top of the polymer brush at high enough grafting density to result in a dense brush [51]. In the arrangement (ii), a brush of diblock copolymer may act as the functional surface capable of moving NPs on their surface [52], of NPs may slide down to the valleys of the surface relief formed on a brush surface [53]. Transitions between different arrangements are possible as well, e.g. the transition (iii) $\rightarrow$ (i) takes place in diblock copolymer brush that acts as stimuli-sensitive and controllable release systems for NPs [54]. Adsorption of NPs into a bulk of a brush, arrangement (iii), leads to a composite material with a synergy of the properties of both constituents. As remarked in Ref. [55], a brush can function as a nanocomposite matrix acting as a reaction chamber for NPs synthesis, a scaffold for immobilization of NPs or as a capping agent for blocking the NPs aggregation leading to new related applications. These, in particular, are: catalysis, sensors, new optical materials, nanoelectrodes, antimicrobial, antiadhesive and nanomagnetic materials, for more details, see Ref. [55].

Most experimental, theoretical and simulation work done in this field, consider undecorated NPs but various types of brushes, e.g. mixed brush, diblock-polymer brush, nanopatterned brush, etc. However, when both the NPs and the brush contain similar LC groups, their interaction became more specific and spatially-resolved, as well as controllable by light if the LC groups are chromophoric, e.g. azobenzenes $[30,47,49,50,56]$. This opens up many possibilities for photo-controlled adsorption/deadsorption of NPs by the LC polymer brush and related applications for controlled drug release, catalysis, organic electronics developments. In this study we analyse the adsoption of NPs on a LC polymer brush in terms of the system structure and dynamics by molecular dynamics computer simulations. The model details are considered in Sec. 2, the results are discussed in Sec. 3, followed by Conclusions. 


\section{The model}

The intrinsic time- and length-scale for the adsorption of decorated NP on brush makes it logical to employ the coarse-grained modelling. It is done by splitting the molecule into beads each representing certain characteristic atomic group. The beads are interacting via effective potentials, typically of rather soft nature. Effective potentials are either derived by parametrization based on the use of simulations of a more detailed model $[57,58]$, or postulated as a simple analytic form with their coefficients found from matching compressibility, viscosity or phase behavior of a certain real system. The best known realisation of the latter scenario is the dissipative particle dynamics approach [59]. Here we use the coarse-grained model for a NP decorated by the LC ligands used in a number of previous studies [35, 39, 40,42,43,60,61], which is based on the earlier works [62-64].

The model NP comprises a large core bead decorated by 12 ligands each containing a short spacer of two beads and a terminal mesogen. As shown in Ref. [35], in poor solvent conditions, such NPs form a nanogel due to the attraction between the mesogens. The properties of a nanogel depend strongly on the decoration pattern on the NP surface. Out of many decoration patterns considered in Ref. [35] we use here the HDG one, characterised by the icosahedral arrangement of ligands on the surface of the core bead. Such arrangement mimics, for instance, the tetrahedral decoration of the fullerene $[65,66]$. In the simulations, it was found to produce only local links between NP in solution but not a single-network nanogel structure, [35] thus enabling diffusion of the NPs and, hence, their possible adsorption on the brush. The brush molecules are of the side-chain architecture containing a backbone of 36 beads and 12 side chains spread uniformly across it, each side-chain contains a two-bead spacer and a terminal mesogen of the same type as in the NPs. The first bead of each backbone is grafted to the bottom surface of the simulation box chosen as the $z=0$ plane, as shown in Fig. 1 .

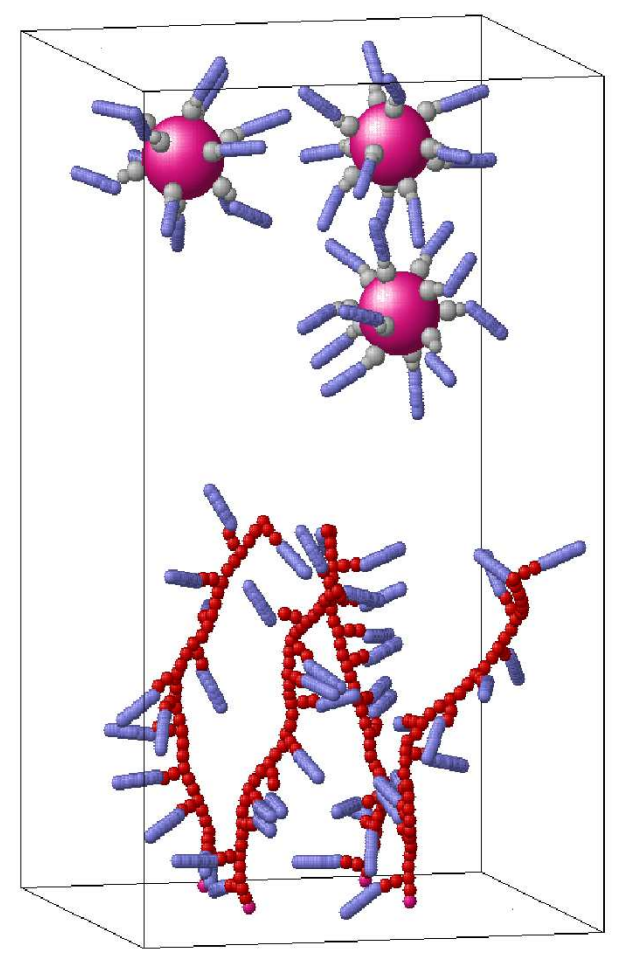

Fig. 1. Illustration of a modelling setup used in this study. The NP core unit is shown as a large pink sphere, ligands contain a spacer shown in gray and terminating LC bead, brush monomers are shown in red with their side chains terminated by the same $\mathrm{KC}$ bead as in NPs.

We will concentrate on the dimensions of the beads and on the details of the effective interactions now. Let $\sigma_{0}$ define the reduced unit of length. Then, the diameters of the spherical beads comprising decorated NP in reduced units are: $\sigma=4.656$ for the core bead, $\sigma=1.357$ for the first and $\sigma=1$ for the second bead of a spacer, based on the earlier estimates from Ref. [62,63]. The mesogens of both decorated NPs and brush molecules are characterised by the diameter of their spherical caps $D=0.815$ and their length-to-breadth ratio of $L / D=3$, based on the dimensions of typical mesogens (e.g. 5CB) in real units. Spherical beads of the brush molecules and that of a solvent are chosen to be of the same diameter $\sigma=1$ as the smaller spacer bead in the NP.

Both types of beads, spherical and spherocylinder, are convex bodies and we use the Kihara type of the non-bonded interaction potential [67]. In this formalism, one assumes the presence of an internal interaction core: the center of the sphere and a line joining the centers of spherical caps, respectively. If $\mathbf{r}_{i j}$ is the vector that connects the centers of $i$ th and $j$ th beads and $\widehat{\mathbf{e}}_{i}$ and $\widehat{\mathbf{e}}_{j}$ - their orientations (for spherocylinder beads only), then the set $\mathbf{q}_{i j}=\left\{\widehat{\mathbf{e}}_{i}, \widehat{\mathbf{e}}_{j}, \mathbf{r}_{i j}\right\}$ fully characterizes the mutual spatial 
position of the $i$ th and $j$ th beads. The shortest distance between the internal cores, $d\left(\mathbf{q}_{i j}\right)$, is evaluated and enters the analytic form for the effective potential with quadratic soft-core repulsion of the type $\sim\left[1-d\left(\mathbf{q}_{i j}\right]^{2}\right.$. As far as our model contains spherical beads of rather different size, application of a usual combination rule $\sigma_{i j}=\left(\sigma_{i}+\sigma_{j}\right) / 2$ will soften significantly the repulsion near the surface of a large sphere (e.g. a core bead). Therefore, we use the shifted form of the interaction potential

$$
V^{\mathrm{SRP}}= \begin{cases}u, & d\left(\mathbf{q}_{i j}\right)<\sigma_{i j}-1, \\ u\left[1-\left[d\left(\mathbf{q}_{i j}\right)-\sigma_{i j}+1\right]\right]^{2}, & \sigma_{i j}-1 \leqslant d\left(\mathbf{q}_{i j}\right) \leqslant \sigma_{i j}, \\ 0, & d\left(\mathbf{q}_{i j}\right)>\sigma_{i j} .\end{cases}
$$

In a special case of $\sigma_{i j}=1$ the shift disappears. The energy prefactor $u$ in Eq. (1) is set to a different value depending on the types of interacting beads. It is expressed in reduced units with the energy scale given by $u_{0}$, characteristic energy of repulsion of two spacer beads. We assume $u=1$ for all pairs not involving the solvent. To mimic poor solvent conditions we set stronger repulsion with $u=2$ between the NP beads and these of a solvent.

In the case of NP with metallic cores, e.g. $\mathrm{Ag}$ and $\mathrm{Au}$, a double electric layer is formed around each surface-stabilized core [30] leading to the stronger core-core repulsion. Following Ref. [35], we use for this type of interaction shifted repulsive $a d$ hoc potential of adjustable range $r_{c}$ with the energy prefactor $u=2$

$$
V_{c}^{\mathrm{SRP}}= \begin{cases}2 u, & r_{i j}<\sigma_{c} \\ 2 u\left[1-\frac{r_{i j}-\sigma_{c}}{r_{c}-\sigma_{c}}\right]^{2}, & \sigma_{c} \leqslant r_{i j} \leqslant r_{c} \\ 0, & r_{i j}>r_{c}\end{cases}
$$

This interaction potential can be interpreted as a primitive model for screened Coulomb potential with a range $r_{c}=11.7$ chosen not to exceed $L_{x} / 2$.

The mesogen-mesogen potential is of the Lintuvuori-Wilson form [64] rewritten for the sake of convenience in reduced units, following Refs. [41,68]

$$
\begin{gathered}
V^{\mathrm{SAP}}= \begin{cases}u\left\{\left[1-\frac{d\left(\mathbf{q}_{i j}\right)}{D}\right]^{2}-\varepsilon^{\prime}\left(\mathbf{q}_{i j}\right)\right\}, & \frac{d\left(\mathbf{q}_{i j}\right)}{D}<1, \\
u\left\{\left[1-\frac{d\left(\mathbf{q}_{i j}\right)}{D}\right]^{2}-\varepsilon^{\prime}\left(\mathbf{q}_{i j}\right)-\frac{1}{4 \varepsilon^{\prime}\left(\mathbf{q}_{i j}\right)}\left[1-\frac{d\left(\mathbf{q}_{i j}\right)}{D}\right]^{4}\right\}, & 1 \leqslant \frac{d\left(\mathbf{q}_{i j}\right)}{D} \leqslant d_{c}, \\
0, & \frac{d\left(\mathbf{q}_{i j}\right)}{D}>d_{c},\end{cases} \\
\varepsilon\left(\mathbf{q}_{i j}\right)=\left\{4\left[U_{a}^{\prime}-5 \varepsilon_{1}^{\prime} P_{2}\left(\widehat{\mathbf{e}}_{i} \cdot \widehat{\mathbf{e}}_{j}\right)-5 \varepsilon_{2}^{\prime}\left(P_{2}\left(\widehat{\mathbf{r}}_{i j} \cdot \widehat{\mathbf{e}}_{i}\right)+P_{2}\left(\widehat{\mathbf{r}}_{i j} \cdot \widehat{\mathbf{e}}_{j}\right)\right)\right]\right\}^{-1}
\end{gathered}
$$

where the dimensionless configuration dependent well depth $\varepsilon^{\prime}\left(\mathbf{q}_{i j}\right)$ is obtained from the requirement that both the potential and its first derivative vanish at $d_{c}$ [64]. Here $\widehat{\mathbf{r}}_{i j}=\mathbf{r}_{i j} / r_{i j}$ is the unit vector along the vector $\mathbf{r}_{i j}, U_{a}^{\prime}=21.43, \varepsilon_{1}^{\prime}=1.714$ and $\varepsilon_{2}^{\prime}=-1.714$ are dimensionless parameters that define the shape of the potential and a chosen to reproduce the "model A" being studied in Ref. [64], $P_{2}(x)=\left(3 x^{2}-1\right) / 2$ is the second Legendre polynomial.

The expression for the bonded interactions

$$
V^{\text {bon }}=\sum_{i=1}^{N_{N P}} V_{N P}^{\text {bon }}+\sum_{i=1}^{N_{B}} V_{B}^{\text {bon }}
$$

includes a sum of the contributions from $N_{N P}$ NPs, each given as

$$
V_{N P}^{\mathrm{bon}}=\sum_{i=1}^{n_{b}} k_{b}\left(l_{i}-l_{0}\right)^{2}+\sum_{i=1}^{n_{a}} k_{a}\left(\theta_{i}-\theta_{0}\right)^{2}+\sum_{i=1}^{n_{a}^{\prime}} k_{a}^{\prime}\left(\theta_{i}-\theta_{0}^{\prime}\right)^{2}+\sum_{i=1}^{n_{z}} k_{z}\left(\zeta_{i}-\zeta_{0}\right)^{2},
$$

Mathematical Modeling and Computing, Vol. 7, No. 2, pp. 207-218 (2020) 
and the same from $N_{B}$ brush molecules, the contribution from a single molecule is

$$
V_{B}^{\text {bon }}=\sum_{i=1}^{m_{b}} k_{b}\left(l_{i}-l_{0}\right)^{2}+\sum_{i=1}^{m_{a}} k_{a}\left(\theta_{i}-\theta_{0}\right)^{2}+\sum_{i=1}^{m_{a}^{\prime \prime}} k_{a}^{\prime \prime}\left(\theta_{i}-\theta_{0}^{\prime \prime}\right)^{2}+\sum_{i=1}^{m_{z}} k_{z}\left(\zeta_{i}-\zeta_{0}\right)^{2} .
$$

Each NP contains $n_{b}=36$ bonds, the number of bonds for each brush molecule is $m_{b}=71$. The bond energy constant is set to $k_{b}=15$ in reduced units and is the same for all bonds. The reference bond lengths are: $l_{0}=3.25$ (core - first spacer bead in NP), $l_{0}=0.784$ (first-to-second spacer bead in NP and all bonds between spherical beads in brush molecule) and $l_{0}=1.87$ (spacer bead - center of a terminal mesogen in both NP and brush molecule). In total three types of pseudo-valent angles are used. The angles of the first type control the amount of stiffness of linear polymer fragments (a backbone, side-chains and ligands) and are characterised by the reference angle $\theta_{0}=\pi$ and the force constant of $k_{a}=0.286 / \mathrm{rad}^{2}$. There are $n_{a}=24$ of them in each NP and $m_{a}=58$ in each brush molecule. The second type of angles is introduced for the NP to keep the icosahedral arrangement of ligands. To this end we introduce the angle [i]-[core]-[k] for each pair $(i, k)$ of vertices forming an edge of the icosahedra, in total $n_{a}^{\prime}=30$ angles. As the result, the icosahedral decoration scaffold keeps its arrangement but is allowed to slide over the surface of the core bead, thus eliminating the need for rotational degrees of freedom for the NPs $[35,69,70]$. These angles are characterised by the reference angle $\theta_{0}^{\prime}=\pi / 3$ and the force constant $k_{a}^{\prime}=0.714 / \mathrm{rad}^{2}$. Finally, the angles of the third type are introduced to keep the side-chains of the brush molecules preferably perpendicular to their host backbones. Each brush molecule contains $m_{a}^{\prime \prime}$ angles of this type characterised by the reference angle $\theta_{0}^{\prime}=\pi / 2$ and the force constant $k_{a}^{\prime \prime}=k_{a}$. Additional terminal angles, $\zeta_{i}$ ensure that the mesogen group is connected properly to the ligand spacer, [71] the parameters of this energy term are given as: $\zeta_{0}=\pi$ and $k_{z}=k_{a}$. The set of angles of all these types also prevent brush from a self-collapse in a poor solvent conditions and, thus, enable them to adsorb the NPs.

\section{Results}

The model system is contained within the simulation box with the dimensions $L_{x}=L_{y}=21.8$ and $L_{z}=54.5$ in reduced units. Periodic boundary conditions are applied along $X$ - and $Y$-axes and elastic reflections are performed when the bead crosses one of $z=0$ or $z=L_{z}$ walls. Dimension $L_{z}$ of the box is chosen due to the requirement that the maximum extension of the brush molecules should not exceed $L_{z} / 2$. We consider the set of various grafting densities of the brush with, respectively $N_{B}=0$, $1,4,9,16,25$ and 36 brush molecules, with their ends grafted at the vertices of a square grid. The number of NPs is fixed at $N_{N P}=20$. This setup is shown in Fig. 1 for the case of $N_{B}=4$ but we reduced the number of NPs there to 3 for the sake of clarity. The rest of the simulation box is filled by the solvent until the total density of the solution reach the value of $0.5 \mathrm{~g} / \mathrm{cm}^{3}$.

The simulations are performed via molecular dynamics in the $N V T$ ensemble at the reduced temperature of $k_{B} T / u_{0}=0.0095$, the same as in Ref. [35], it corresponds to $T=480 \mathrm{~K}$ in Refs. [39,40,43,60]. The temperature control is provided by means of velocity rescaling. Due to soft nature of all interaction potentials, the time-step of the simulations is chosen equal to be around $20 \mathrm{fs}$ in real units.

For each number of brush molecules, $N_{B}$, we perform five "adsorption" runs, each of duration 20ns. During the adsorption runs, the mesogens attract each other and form physical crosslinks. Due to the presence of them both in the NPs ligands and in the brush, the aggregation of the NPs in the bulk part of the simulation box and their adsorption on the brush will compete. The result of the competition is expected to depend on the grafting density, i.e. the number $N_{B}$ of brush molecules. Each such run is preceded by the "refreshing" equilibration run of the same duration, during which we switch off the attraction between the mesogens, i.e. the repulsive interaction potential (1) is used instead of the attractive one (3). This eliminates aggregation of NPs, as well as their adsorption on the brush an also 
allows them to diffuse throughout the simulation box. System properties in the adsorption regime are evaluated by averaging the data obtained during the last $4 \mathrm{~ns}$ of each adsorption run.
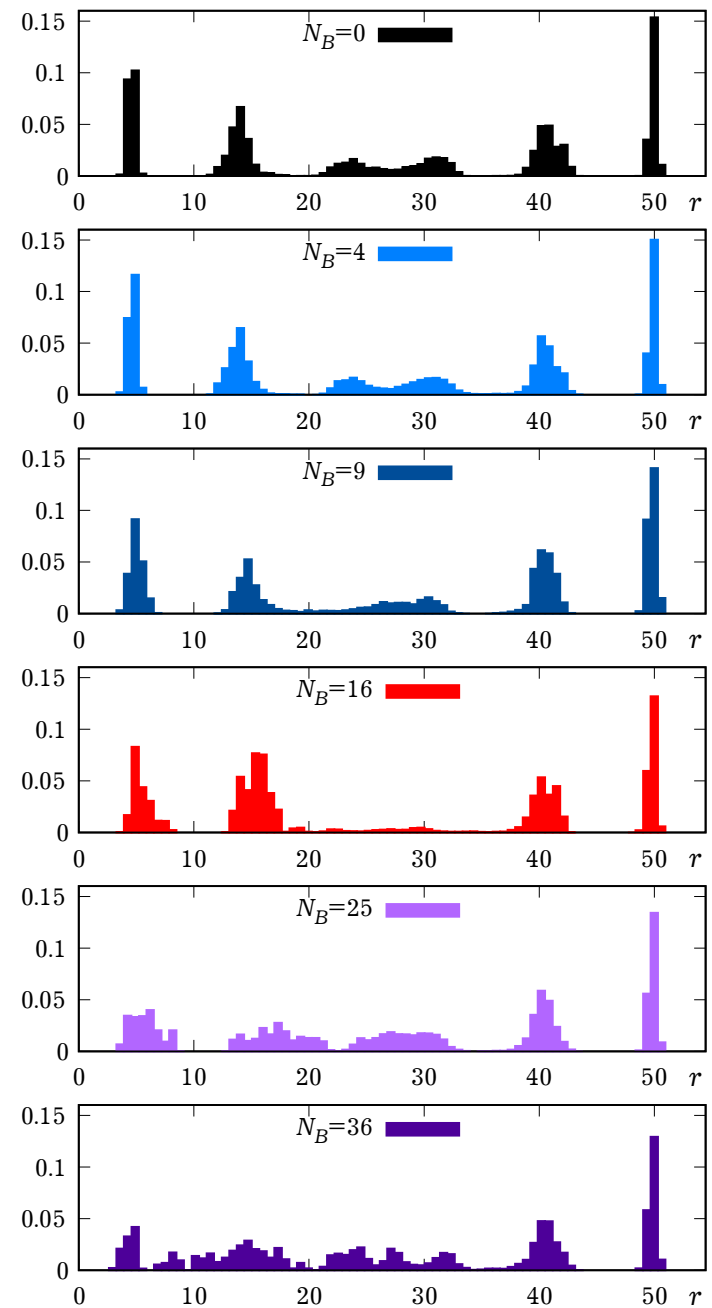

Fig. 2. Histograms for the number density profiles $n_{N P}(z)$ for the NP cores along the $Z$-axis at various number $N_{B}$ of brush molecules (indicated in each plot).

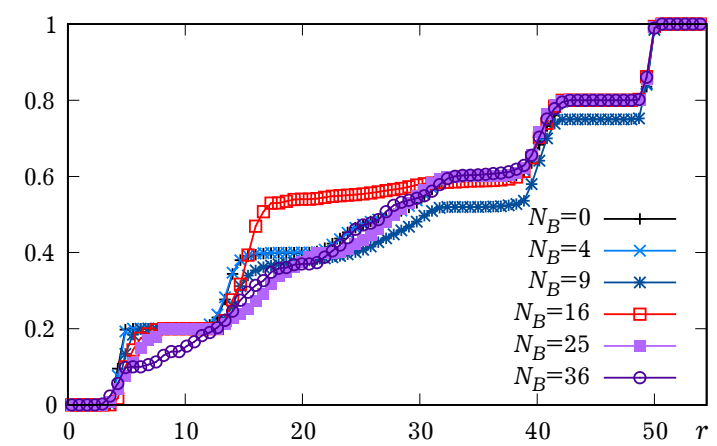

Fig. 3. Cumulative number density $c_{N P}(z)$ for the NP cores along the $Z$-axis at various number $N_{B}$ of brush molecules (indicated in each plot). in Fig. 3. At $N_{B}=0,4,9$ one observes a step-like dependence with $z$ with five plateau, each plateau emerging after the following layer of NPs being incorporated into the interval of $0-z$. At $N_{B}=16$, the second and third plateau merge into a single large one, indicating adsorption of NPs into the brush
Let us examine first the number density profiles for the NP cores along the $Z$-axis

$$
n_{N P}(z)=\frac{N_{N P}(z)}{N_{N P}}
$$

where $N_{N P}(z)$ is the number of NPs such as their core beads are at the separation $z$ from the $z=0$ wall. To allow easy comparison of $n_{N P}(z)$ obtained at various $N_{B}$, they are presented as a stack of histograms, see Fig. 2. With no brush molecules present, $N_{B}=0$, one observes the effect of layering of NPs near each wall with the peak heights decaying and their widths increasing as one moves towards the middle region of the box $(20<z<35)$. Density profile at $N_{B}=4$ indicates no visible changes, whereas at $N_{B}=9$ there is a subtle change in the middle region of the box. At yet higher number $N_{B}=16$ of brush chains, the profile in the middle region of the box disappears, whereas the width of the second peak, as counted from the $z=0$ wall, increases. This indicates adsorption of the NP from the middle region of the box into the upper part of the brush. With the further increase of $N_{B}, N_{B}=25,36$ two effects are observed: (i) $n_{N P}(z)$ became smeared in the brush region $z<20$, and (ii) some NPs escape from the brush now into the middle region of the box. This may indicate that the adsorption of NPs is hindered by too high density of the brush that literally "push out" the superfluous NPs.

The presence of an optimal value of $N_{B} \approx 16$ for adsorption of NPs in a brush in this particular model is further confirmed by evaluation of the cumulative number density of NPs

$$
c_{N P}(z)=\sum_{z_{i}<z} n_{N P}\left(z_{i}\right)
$$

which has a meaning of a fraction of NPs with their core beads found at the separation less than $z$ from the bottom wall (the property that can be associated with the uniaxial coordination number in the theory of liquids). The plots for $c_{N P}(z)$ obtained at different $N_{B}$ are shown 
already at $z \approx 18$. With the increase of $N_{B}$ up to 36 , the steps for $c_{N P}(z)$ disappear for all $z<30$ and are found again only in the bulk part of the simulation box, $z>30$.

The changes undergone by the arrangement of NPs and the structure of a brush upon the increase of $N_{B}$ from 4 through 16 up to 36 , are visualised in a series of snapshots Fig. 4 . One should note a gradual increase of the effective brush height (about $0.25 L_{z}, 0.4 L_{z}$ and $0.5 L_{z}$, respectively) and upward straightening of its individual molecules, characteristic to the transition to the dense brush regime. These two factors, alongside with the sheer increase of the number of mesogens within a brush, must be responsible for the enhanced adsorption of NPs in a lower brush density regime, culminating at $N_{B} \approx 16$. With the further increase of $N_{B}$, two other factors must be dominating, namely: a lack of an accessible volume for NPs within a brush and a self-assembly of the brush molecules into some ordered lamellar-like morphology (see, Fig. 4 on the right). These factors are most likely responsible for hampering the adsorption of the NPs.

We will proceed now to the short-time dynamics of the NPs depending on their separation $z$ from the bottom wall and the number of $N_{B}$ of brush molecules. It is examined via the average mean square displacements (MSDs) of the core beads evaluated at given time interval $\Delta t$ and within each bin that includes $z$

$$
\begin{aligned}
M S D(\Delta t, z) & =\left.\left\langle\left[\mathbf{r}_{i}\left(t_{2}\right)-\mathbf{r}_{i}\left(t_{1}\right)\right]^{2}\right\rangle\right|_{z_{i}\left(t_{1}\right)=z} \\
t_{2}-t_{1} & =\Delta t
\end{aligned}
$$

Here we identify the MSD host bin via the position of the core bead at the initial time instance $t_{1}$. The averaging is performed over the core beads of all NPs and over all available initial time instances $t_{1}$ within the averaging intervals of adsorption runs, as defined above. The result is shown in Fig. 5 as a series of overlapping plots build for an equidistant time intervals $\Delta t$ for four cases of $N_{B}=0,4,16$ and 36 . This allows for monitoring the increase of MSD with $\Delta t$ at each separation $z$ from the bottom wall.

Let us consider the case with no brush, $N_{B}=$ 0 , first. The MSDs are arranged in stripes following layering arrangement of NPs shown earlier in Fig. 2. At the same $\Delta t$, the height of each stripe
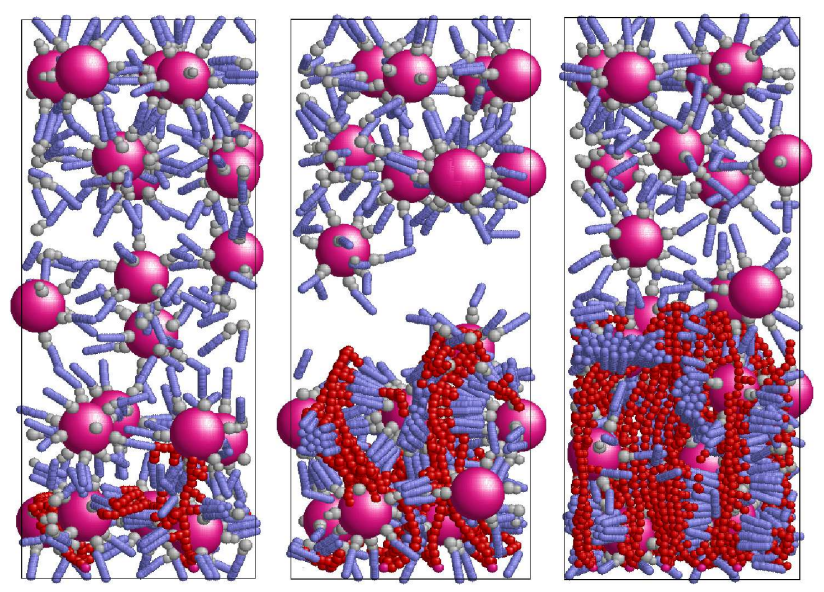

Fig. 4. Snapshots for the brush and the NPs system at various number of brush molecules $N_{B}$. The $N_{B}=4$ case is shown on the left, $N_{B}=16$ - in the middle, $N_{B}=36-$ on the right.
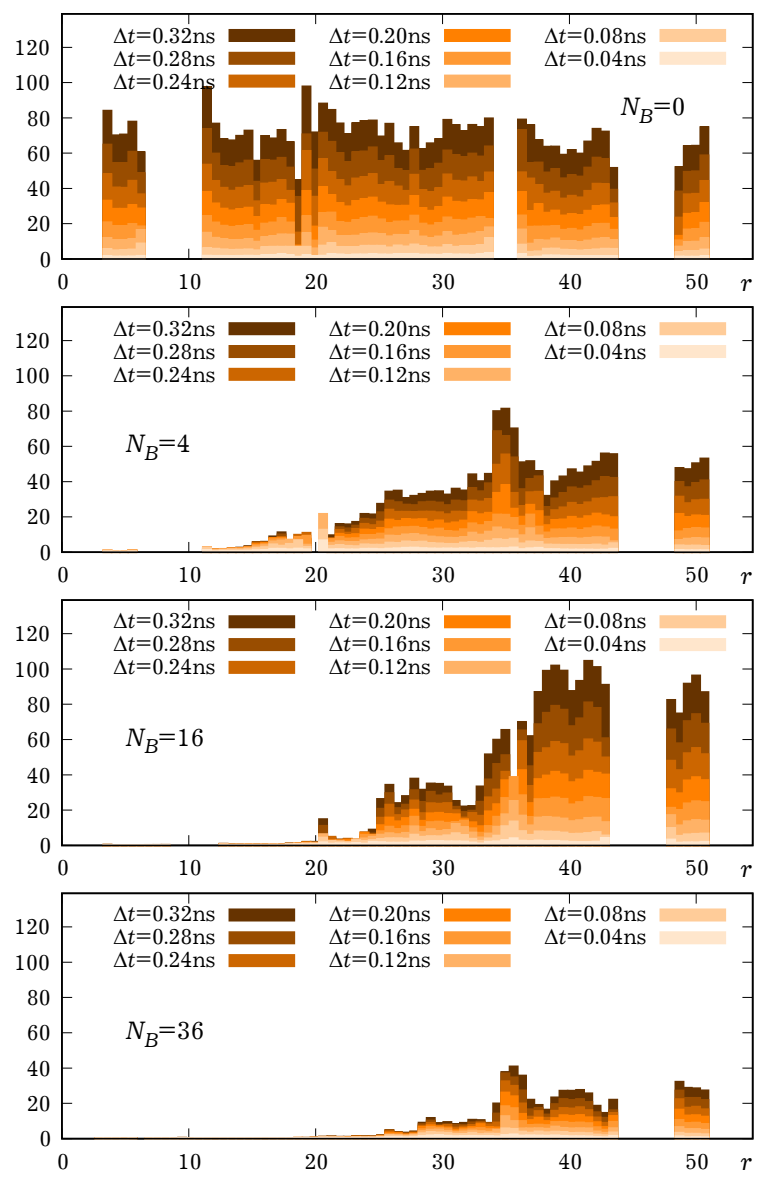

Fig. 5. The average mean square displacements $M S D(\Delta t, z)$ of the core beads at equidistant time intervals $\Delta t$ binned along the $Z$-axis. The number $N_{B}$ of brush molecules is indicated in each plot. is found approximately equal. The increase of MSD upon increase of $\Delta t$ in each $z$-bin is getting approximately linear for $\Delta t>0.24 \mathrm{~ns}$ indicating the start of diffusive regime. Now we will switch to the case 
of $N_{B}=4$. One should recall, that at this low brush density regime, no changes in NPs arrangement is detected (see, Fig. 2). This small number of brush molecules is, however, sufficient to immobilise the NPs within the region of a brush $z<0.25 L z \approx 14$. Similar effect is observed for the rest cases of $N_{B}=16$ and 36, where the adsorbed NPs are found immobilised within the approximate height of the brush, $z<0.4 L z \approx 22$ and $z<0.5 L z \approx 27$, respectively. At the highest value of $N_{B}=36$, the dynamics of NPs in the bulk part of the box is seriously reduced, too. The reason can bee seen in the increase of the local density of NPs there, due to their expulsion from the brush region.

\section{Conclusions}

In this study we considered the system of NPs, decorated by the LC groups, along with the LC polymer brush, built of polymer chains with side-chain architecture. The latter are grafted to the fixed points on the bottom wall of the simulation box and form so called functional surface. The same type of the LC groups is used in both NPs and in the brush molecules, leading to: (i) aggregation of NPs in a bulk part of the simulation box, (ii) self-collapse of the LC polymer brush, and (iii) adsorption of NPs on the brush. Our aim was to study the prevalence of the particular effect depending on the brush density.

After conducting computer simulations and analyzing NP cores density profiles $n_{N P}(z)$ along the $Z$-axis at various number $N_{B}$ of brush molecules, it was found that for $N_{B}=0, N_{B}=4$ and $N_{B}=9$ NPs accumulate near each wall with the peak heights decaying and their widths increasing as one moves towards the middle region of the box. The value of $N_{B}=16$ is the most optimal one, because in this case one observes the most intense NPs adsorption on the upper part of polymer brush. With further increase of $N_{B}$, namely $N_{B}=25,36$, density profile values demonstrate, that NPs are more and more evenly distributed in the brush region. However some of them are, on the contrary, shifted from the brush to the middle region of a simulation box. It means, that the density of brush molecules becomes too high, which prevents NPs adsorption and literally pushes out "extra" NPs out of it.

The presence of an optimal value of $N_{B} \approx 16$ for adsorption of NPs in a brush in this particular model is further confirmed by evaluation of the cumulative number density of NPs. In the case of a gradual increase of both $N_{B}$ and effective brush height (about $0.25 L_{z}, 0.4 L_{z}$ and $0.5 L_{z}$, respectively), an adsorption stops at $N_{B} \approx 16$ due to lack of an accessible volume for NPs within a brush and a self-assembly of the brush molecules into ordered lamellar-like morphology.

The brush density value $N_{B} \approx 16$ is also confirmed as most optimal one for NPs adsorption by analyzing short-time dynamics of the NPs. It is examined via the average mean square displacements (MSDs) of the core beads evaluated at given time interval $\Delta t$ and within each bin that includes $z$ (distance to the bottom wall of the simulation box). One should note, that both for $N_{B}=16$ and $N_{B}=36$ adsorbed NPs are located approximately on the height of the brush, but for latter one, the dynamics of NPs in the bulk part of the box is seriously reduced. The reason for this can be an increase of the local density of NPs there, due to their expulsion from the brush region.

The study can be continued to provide more insight on the properties of the LC brush itself, depending on its density, chains flexibility and the details of molecular architecture. Other possible extension is to consider various decoration patterns of ligands on the NPs surface and their role in the interplay between the aggregation and adsorption depending on the system setup. One also would benefit of considering the larger system sizes and performing averaging over a larger number of simulation runs.

\section{Acknowledgements}

J.M.I. is grateful to J.-U.Sommer for stimulating discussions. 
[1] Lu Y., Liu J. Catalyst-functionalized nanomaterials. Wiley Interdisciplinary Reviews: Nanomedicine and Nanobiotechnology. 1 (1), 35-46 (2008).

[2] Pawar A. B., Kretzschmar I. Fabrication, Assembly, and Application of Patchy Particles. Macromolecular Rapid Communications. 31, 150-168 (2010).

[3] LiZ.W., Zhu Y.L., LuZ.Y., SunZ. Y. A versatile model for soft patchy particles with various patch arrangements. Soft Matter. 12 (3), 741-749 (2016).

[4] Toshima N. Metal Nanoparticles for Catalysis. ChemInform. 35 (34), 79-96 (2004).

[5] Katz E., Shipway A. N., Willner I. Chemically Functionalized Metal Nanoparticles. Sythesis, Properties and Applications, in L. M. Liz-Marzan, P.V. Kamat, editors. Nanoscale Materials, chapter 2, pp. 5-78, Nanoscale Materials (2003).

[6] Amendola V., Pilot R., Frasconi M., Maragò O. M., Iatì M. A. Surface plasmon resonance in gold nanoparticles: a review. Journal of Physics: Condensed Matter. 29 (20), 203002 (2017).

[7] Alshammari A., Kalevaru V. N. Supported Gold Nanoparticles as Promising Catalysts, in Catalytic Application of Nano-Gold Catalysts. InTech (2016).

[8] Choueiri R. M., Galati E., Thérien-Aubin H., Klinkova A., Larin E. M., Querejeta-Fernández A., Han L., Xin H. L., Gang O., Zhulina E. B., Rubinstein M., Kumacheva E. Surface patterning of nanoparticles with polymer patches. Nature. 538 (7623), 79-83 (2016).

[9] Kumar S., Pal S. K., Lakshminarayanan V. Discotic-Decorated Gold Nanoparticles. Molecular Crystals and Liquid Crystals. 434 (1), 251/[579]-258/[586] (2005).

[10] Sergeyev S., Pisula W., Geerts Y.H. Discotic liquid crystals: a new generation of organic semiconductors. Chemical Society Reviews. 36 (12), 1902 (2007).

[11] Kumar S. Discotic liquid crystal-nanoparticle hybrid systems. NPG Asia Materials. 6 (1), e82 (2014).

[12] Yan W., Seifermann S. M., Pierrat P., Bräse S. Synthesis of highly functionalized $\mathrm{C}_{6} 0$ fullerene derivatives and their applications in material and life sciences. Organic \& Biomolecular Chemistry. 13 (1), 25-54 (2015).

[13] De D. S., Flores-Livas J. A., Saha S., Genovese L., Goedecker S. Stable structures of exohedrally decorated $\mathrm{C}_{60}$-fullerenes. Carbon. 129, 847-853 (2018).

[14] Zhang G., Chen Q., Zhang Y., Kong L., Tao X., Lu H., Tian Y., Yang J. Bulky group functionalized porphyrin and its Zn (II) complex with high emission in aggregation. Inorganic Chemistry Communications. 46, 85-88 (2014).

[15] Hvolbæk B., Janssens T. V., Clausen B. S., Falsig H., Christensen C. H., Nørskov J. K. Catalytic activity of Au nanoparticles. Nano Today. 2 (4), 14-18 (2007).

[16] Thompson D. T. Using gold nanoparticles for catalysis. Nano Today. 2 (4), 40-43 (2007).

[17] Yeh Y.C., Creran B., Rotello V.M. Gold nanoparticles: preparation, properties, and applications in bionanotechnology. Nanoscale. 4 (6), 1871-1880 (2012).

[18] Das M., Shim K. H., AnS.S. A., Yi D. K. Review on gold nanoparticles and their applications. Toxicology and Environmental Health Sciences. 3 (4), 193-205 (2011).

[19] Pranami G. Understanding nanoparticle aggregation. Ph.D. thesis, Graduate College, Iowa State University, 10859 (2009).

[20] Gerth M., Voets I. K. Molecular control over colloidal assembly. Chemical Communications. 53 (32), 44144428 (2017).

[21] Yang Y., Matsubara S., Nogami M., Shi J. Controlling the aggregation behavior of gold nanoparticles. Materials Science and Engineering: B. 140 (3), 172-176 (2007).

[22] Chegel V., Rachkov O., Lopatynskyi A., Ishihara S., Yanchuk I., Nemoto Y., Hill J. P., Ariga K. Gold Nanoparticles Aggregation: Drastic Effect of Cooperative Functionalities in a Single Molecular Conjugate. The Journal of Physical Chemistry C. 116 (4), 2683-2690 (2012).

[23] Gao B., Rozin M. J., Tao A. R. Plasmonic nanocomposites: polymer-guided strategies for assembling metal nanoparticles. Nanoscale. 5 (13), 5677 (2013).

[24] Yi C., Zhang S., Webb K. T., Nie Z. Anisotropic Self-Assembly of Hairy Inorganic Nanoparticles. Accounts of Chemical Research. 50 (1), 12-21 (2016). 
[25] Rotello V. M. Interfacing Inorganic Nanoparticles with Biology. Bioconjugate Chemistry. 28 (1), 1-2 (2017).

[26] Posel Z., Posocco P., Lísal M., Fermeglia M., Pricl S. Highly grafted polystyrene/polyvinylpyridine polymer gold nanoparticles in a good solvent: effects of chain length and composition. Soft Matter. 12 (15), 3600-3611 (2016).

[27] Klajn R., Bishop K. J. M., Grzybowski B. A. Light-controlled self-assembly of reversible and irreversible nanoparticle suprastructures. Proceedings of the National Academy of Sciences. 104 (25), 10305-10309 (2007).

[28] Kawai T., Sumi A., Morita C., Kondo T. Preparation and photocoagulation in chloroform of Au nanoparticles capped with azobenzene-derivatized alkanesulfides. Colloids and Surfaces A: Physicochemical and Engineering Aspects. 321 (1-3), 308-312 (2008).

[29] Wei Y., Han S., Kim J., Soh S., Grzybowski B. A. Photoswitchable Catalysis Mediated by Dynamic Aggregation of Nanoparticles. Journal of the American Chemical Society. 132 (32), 11018-11020 (2010).

[30] Lysyakova L., Lomadze N., Neher D., Maximova K., Kabashin A. V., Santer S. Light-Tunable Plasmonic Nanoarchitectures Using Gold Nanoparticle-Azobenzene-Containing Cationic Surfactant Complexes. The Journal of Physical Chemistry C. 119 (7), 3762-3770 (2015).

[31] Freund H. J., Libuda J., Bäumer M., Risse T., Carlsson A. Cluster, facets, and edges: Site-dependent selective chemistry on model catalysts. The Chemical Record. 3 (3), 181-201 (2003).

[32] Sperling R. A., Parak W. J. Surface modification, functionalization and bioconjugation of colloidal inorganic nanoparticles. Philosophical Transactions of the Royal Society A: Mathematical, Physical and Engineering Sciences. 368 (1915), 1333-1383 (2010).

[33] Gröschel A.H., Walther A., Löbling T. I., Schacher F.H., Schmalz H., Müller A.H. E. Guided hierarchical co-assembly of soft patchy nanoparticles. Nature. 503 (7475), 247-251 (2013).

[34] Zhou Y., Ma X., Zhang L., Lin J. Directed assembly of functionalized nanoparticles with amphiphilic diblock copolymers. Physical Chemistry Chemical Physics. 19 (28), 18757-18766 (2017).

[35] Ilnytskyi J. M., Slyusarchuk A., SokołowskiS. Gelation of patchy ligand shell nanoparticles decorated by liquid-crystalline ligands: computer simulation study. Soft Matter. 14 (19), 3799-3810 (2018).

[36] Nandivada H., Ross A. M., Lahann J. Stimuli-responsive monolayers for biotechnology. Progress in Polymer Science. 35 (1-2), 141-154 (2010).

[37] Hou L., Wang L., Zhang N., Xie Z., Dong D. Polymer brushes on metal-organic frameworks by UV-induced photopolymerization. Polymer Chemistry. 7 (37), 5828-5834 (2016).

[38] Feng C., Huang X. Polymer Brushes: Efficient Synthesis and Applications. Accounts of Chemical Research. 51 (9), 2314-2323 (2018).

[39] Ilnytskyi J., Lintuvuori J., Wilson M. R. Simulation of bulk phases formed by polyphilic liquid crystal dendrimers. Condensed Matter Physics. 13 (3), 33001 (2010).

[40] Ilnytskyi J. Relation between the grafting density of liquid crystal macromolecule and the symmetry of self-assembled bulk phase: coarse-grained molecular dynamics study. Condensed Matter Physics. 16 (4), 43004 (2013).

[41] Ilnytskyi J. M., Trokhymchuk A., Schoen M. Topological defects around a spherical nanoparticle in nematic liquid crystal: Coarse-grained molecular dynamics simulations. The Journal of Chemical Physics. 141 (11), 114903 (2014).

[42] Ilnytskyi J., Slyusarchuk A., Saphiannikova M. Photo-controllable percolation of decorated nanoparticles in a nanopore: molecular dynamics simulation study. Mathematical Modeling and Computing. 3 (1), 33-42 (2016).

[43] Ilnytskyi J. M., Slyusarchuk A., Saphiannikova M. Photocontrollable Self-Assembly of AzobenzeneDecorated Nanoparticles in Bulk: Computer Simulation Study. Macromolecules. 49 (23), 9272-9282 (2016).

[44] Williams D. R. M., Halperin A. Liquid-crystalline polymers in good nematic solvents: free chains, mushrooms, and brushes. Macromolecules. 26 (16), 4208-4219 (1993).

[45] Shusaku N. Inducing Planar Orientation in Side-Chain Liquid-Crystalline Polymer Systems via Interfacial Control. The Chemical Record. 16 (1), 378-392 (2016). 
[46] Peng B., Johannsmann D., Ruhe J. Polymer Brushes with Liquid Crystalline Side Chains. Macromolecules. 32 (20), 6759-6766 (1999).

[47] Uekusa T., Nagano S., Seki T. Unique Molecular Orientation in a Smectic Liquid Crystalline Polymer Film Attained by Surface-Initiated Graft Polymerization. Langmuir. 23 (8), 4642-4645 (2007).

[48] Mukai K., Hara M., Nagano S., Seki T. High-Density Liquid-Crystalline Polymer Brushes Formed by Surface Segregation and Self-Assembly. Angewandte Chemie International Edition. 55 (45), 14028-14032 (2016).

[49] Santer S. Remote control of soft nano-objects by light using azobenzene containing surfactants. Journal of Physics D: Applied Physics. 51 (1), 013002 (2017).

[50] Kopyshev A., Galvin C. J., Patil R. R., Genzer J., Lomadze N., Feldmann D., Zakrevski J., Santer S. LightInduced Reversible Change of Roughness and Thickness of Photosensitive Polymer Brushes. ACS Applied Materials \& Interfaces. 8 (29), 19175-19184 (2016).

[51] Cheng S., Stevens M. J., Grest G. S. Ordering nanoparticles with polymer brushes. The Journal of Chemical Physics. 147 (22), 224901 (2017).

[52] Santer S., Rühe J. Motion of nano-objects on polymer brushes. Polymer. 45 (25), 8279-8297 (2004).

[53] Loebner S., Jelken J., Yadavalli N., Sava E., Hurduc N., Santer S. Motion of Adsorbed Nano-Particles on Azobenzene Containing Polymer Films. Molecules. 21 (12), 1663 (2016).

[54] Kumar S., Dory Y.L., Lepage M., Zhao Y. Surface-Grafted Stimuli-Responsive Block Copolymer Brushes for the Thermo-, Photo- and pH-Sensitive Release of Dye Molecules. Macromolecules. 44 (18), 7385-7393 (2011).

[55] Nie G., Li G., Wang L., Zhang X. Nanocomposites of polymer brush and inorganic nanoparticles: preparation, characterization and application. Polymer Chemistry. 7 (4), 753-769 (2016).

[56] Jelken J., Santer S. Light induced reversible structuring of photosensitive polymer films. RSC Advances. 9 (35), 20295-20305 (2019).

[57] Voth G. editor. Coarse-Graining of Condensed Phase and Biomolecular Systems. CRC Press (2008).

[58] RühleV., Junghans C., Lukyanov A., Kremer K., Andrienko D. Versatile Object-Oriented Toolkit for Coarse-Graining Applications. Journal of Chemical Theory and Computation. 5 (12), 3211-3223 (2009).

[59] Steiner T. Dissipative Particle Dynamics: Simulation of Microfluidic Systems with Fluid Particle Methods on High Performance Computers (Microfluidics and Nanofluidics. Shaker Verlag GmbH, Germany (2009).

[60] Slyusarchuk A., Ilnytskyi J. Novel morphologies for laterally decorated metaparticles: molecular dynamics simulation. Condensed Matter Physics. 17 (4), 44001 (2014).

[61] Ilnytskyi J. M. Photo-Controllable Networks in Macromolecular Solutions and Blends, in Y. Holovatch, editor. Order, Disorder and Criticality. 5, 227-269, World Scientific (2017).

[62] Hughes Z. E., Wilson M. R., Stimson L. M. Coarse-grained simulation studies of a liquid crystal dendrimer: towards computational predictions of nanoscale structure through microphase separation. Soft Matter. 1 (6), $436(2005)$.

[63] Hughes Z. E., Stimson L. M., Slim H., Lintuvuori J. S., Ilnytskyi J. M., Wilson M. R. An investigation of softcore potentials for the simulation of mesogenic molecules and molecules composed of rigid and flexible segments. Computer Physics Communications. 178 (10), 724-731 (2008).

[64] LintuvuoriJ.S., Wilson M. R. A new anisotropic soft-core model for the simulation of liquid crystal mesophases. The Journal of Chemical Physics. 128 (4), 044906 (2008).

[65] Fowler P. W., Quinn C. M., Redmond D. B. Decorated fullerenes and model structures for water clusters. The Journal of Chemical Physics. 95 (10), 7678-7681 (1991).

[66] Vukićević R., Beuermann S. Fullerenes Decorated with Poly(vinylidene fluoride). Macromolecules. 44 (8), 2597-2603 (2011).

[67] Kihara T. Convex Molecules in Gaseous and Crystalline States, in Advances in Chemical Physics, pp. 147188, Wiley-Blackwell (1963).

[68] Ilnytskyi J. M., Saphiannikova M. Reorientation Dynamics of Chromophores in Photosensitive Polymers by Means of Coarse-Grained Modeling. ChemPhysChem. 16 (15), 3180-3189 (2015).

[69] Baran E., Sokołowski S. A comparison of molecular dynamics results for two models of nanoparticles with fixed and mobile ligands in two-dimensions. Applied Surface Science. 396, 1343-1351 (2017). 
[70] Baran L., Sokołowski S. Effective interactions between a pair of particles modified with tethered chains. The Journal of Chemical Physics. 147 (4), 044903 (2017).

[71] Wilson M. R., Ilnytskyi J. M., Stimson L. M. Computer simulations of a liquid crystalline dendrimer in liquid crystalline solvents. The Journal of Chemical Physics. 119 (6), 3509 (2003).

\title{
Адсорбція функціоналізованих наночастинок на рідкокристалічній полімерній щітці: дослідження методом молекулярної динаміки
}

\author{
Слюсарчук А. Ю. ${ }^{1}$, Яремчук Д. Л. ${ }^{2}$, Ільницький Я. М. ${ }^{1,2}$ \\ ${ }^{1}$ Національний університет "Львівсъка політехніка", \\ вул. С. Бандери, 12, 79013, Львів, Україна \\ ${ }^{2}$ Інститут фізики конденсованих систем НАН Украӥни, \\ вул. Свенціиьвкого, 1, 79011, Львів, Україна
}

\begin{abstract}
Розглянуто розчин наночастинок у порі, одна із стінок якої є рідкокристалічною полімерною щіткою. Як ліганди наночастинок, так і бічні ланцюжки молекул щітки містять рідкокристалічні групи того ж типу. Дослідження виконано шляхом комп'ютерного моделювання методом молекулярної динаміки. В обох випадках, за низької та високої густини щітки, переважає агрегація між парами наночастинок в об'ємі та між молекулами щітки. Проте, знайдено специфічне значення густини щітки, за якого адсорбція наночастинок на щітці переважає їх агрегацію в об'ємі. Для всіх розглянутих випадків побудовано набори профілів густини та проаналізовано динамічні властивості наночастинок.
\end{abstract}

Ключові слова: наночастинки, рідкі кристали, полімерні щітки, адсорбція. 